\title{
Use of Cantilever Mechanics for Impacted Teeth: Case Series
}

\author{
Sergio Paduano ${ }^{1, *}$, Gianrico Spagnuolo ${ }^{2}$, Gerardo Franzese $^{2}$, Gioacchino Pellegrino $^{2}$, Rosa Valletta $^{2}$ \\ and Iacopo Cioffi ${ }^{2}$
}

\author{
${ }^{1}$ Department of Health Sciences - University Magna Graecia Catanzaro, Italy \\ ${ }^{2}$ Department of Neuroscience, Section of Orthodontics, University of Naples Federico II, Italy
}

\begin{abstract}
This paper describes the orthodontic treatment, and the biomechanics of cantilevers for the impaction of permanent teeth in youngs, adolescents, and adults. In these case series, multibracket straightwire fixed appliances, together with cantilever mechanics, were used to treat the impaired occlusion.
\end{abstract}

Keywords: Tooth impaction, cantilever mechanics, orthodontic case series.

\section{INTRODUCTION}

The impaction of maxillary and mandibular canines is frequently seen in clinical practice [1-3]. It has been suggested that the frequency of palatal impaction of maxillary canines is about five times greater than labial impactions. Furthermore, canine impaction occurs more frequently unilaterally than bilaterally. The aetiology of canine impaction is generally related to the upper dental arch length deficiency, or to the position of the germ of the tooth, that could be very cranial. Mechanical factors have also been proposed to be related to the impaction of a canine. For instance, the premature loss of deciduous canines or their prolonged retention could be associated to mechanical impingement of permanent canine eruption. This can also be the consequence of displacement of lateral incisors or to the presence of impacted teeth or odontomas [1,2].

Orthodontic recovery of impacted teeth can be performed by using cantilever mechanics. Indeed, a two-tooth system with a one-couple can be used to obtain an extrusive force to the canine and an intrusive force to the molar. One-couple orthodontic appliances can increase the predictability of tooth movement, reduce the need of appliance reactivation and the occurrence of possible intra-arch unwanted side effects [4].

Tooth transposition is an anomaly of eruption defined as the positional interchange of two neighboring teeth or the development or eruption of a tooth in a position normally occupied by a non-neighboring tooth [5]. It is a rare phenomenon $(0.33 \%)$, and its occurrence seems to be not related to specific gender differences. It occurs more unilaterally than bilaterally [6].

\footnotetext{
*Address correspondence to this author at the Department of Health Sciences, University of Catanzaro Magna Graecia, Viale Europa Località Germaneto - Catanzaro - Italy; Tel: +39 0961-3694001;
}

Fax: +39 0961-3694001; E-mail: paduano@unicz.it
The orthodontic management of these difficult cases requires the use of specific orthodontic mechanics, which could allow a perfect three dimensional control of tooth movement in order to reduce possible side effects.

We present the cases of a young, an adult, and an adolescent patient with impacted teeth and tooth transposition. Multibracket straightwire fixed appliances, together with cantilever mechanics, were used to treat the impaired occlusion.

\section{CASE PRESENTATION - CASE 1 - WOMAN, 19 YEARS OLD}

\section{Diagnosis and Treatment Plan}

The extraoral and intraoral photographs of the patient are reported in Fig. (1) and Fig. (2). The patient presented this objective problem list:

- $\quad$ Class I malocclusion;

- $\quad$ Presence of the deciduous maxillary right canine, and impaction of the permanent canine

- Impaction of the mandibular left lateral incisor and transposition with canine

- $\quad$ Arch length discrepancies

- Spacings

- $\quad$ Severe brachifacial typology

The patient did not present signs or symptoms of temporomandibular disorders according to Resaerch Diagnostic Criteria for temporomandibular disorders (RDC/TMD) [7]. Nonetheless, the patient reported frequent oral parafuctions, such as clenching, that have been suggested to be related to muscle pain $[8,9]$.

The cephalometric evaluation highlighted a brachifacial typology with a sagittal skeletal relationship of Class I (Fig. 3). 

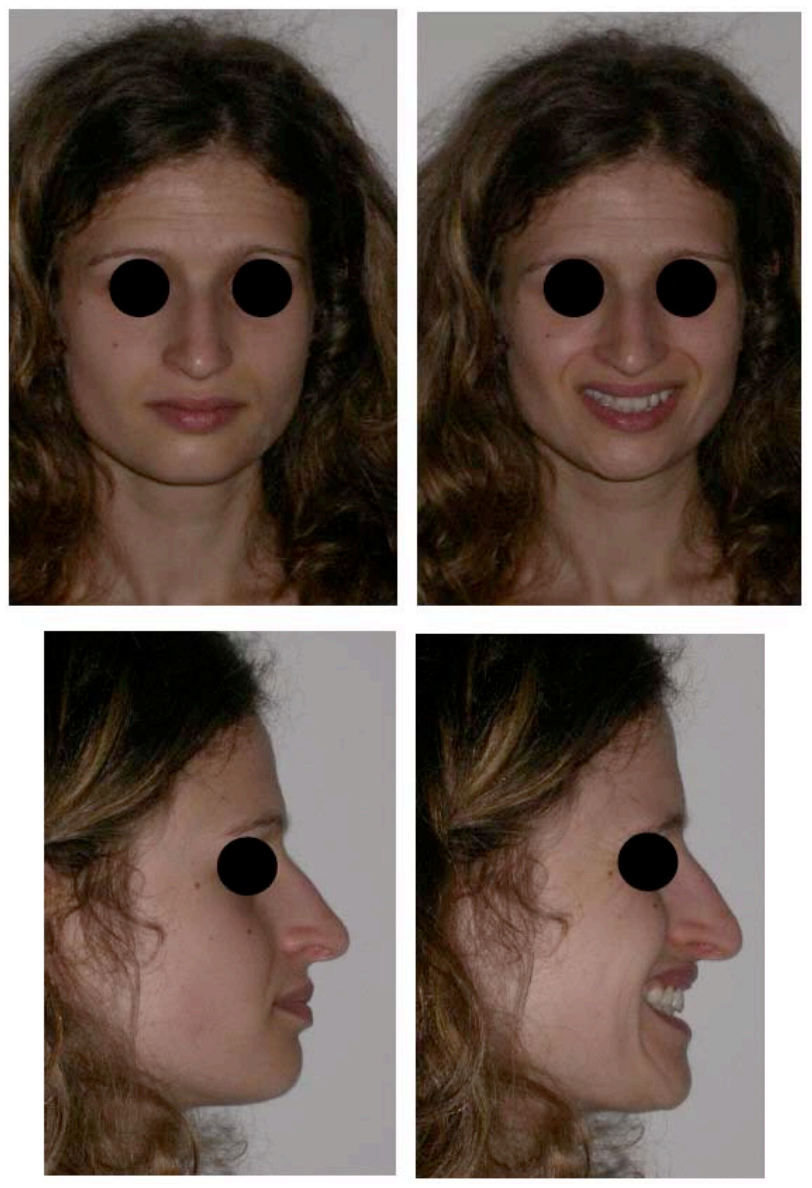

Fig. (1). Extra oral photographs before treatment. Frontal view: no evident asymmetry with good head posture. Upper dental midline is aligned with the facial one. Lateral view: No labial incompetence was present.
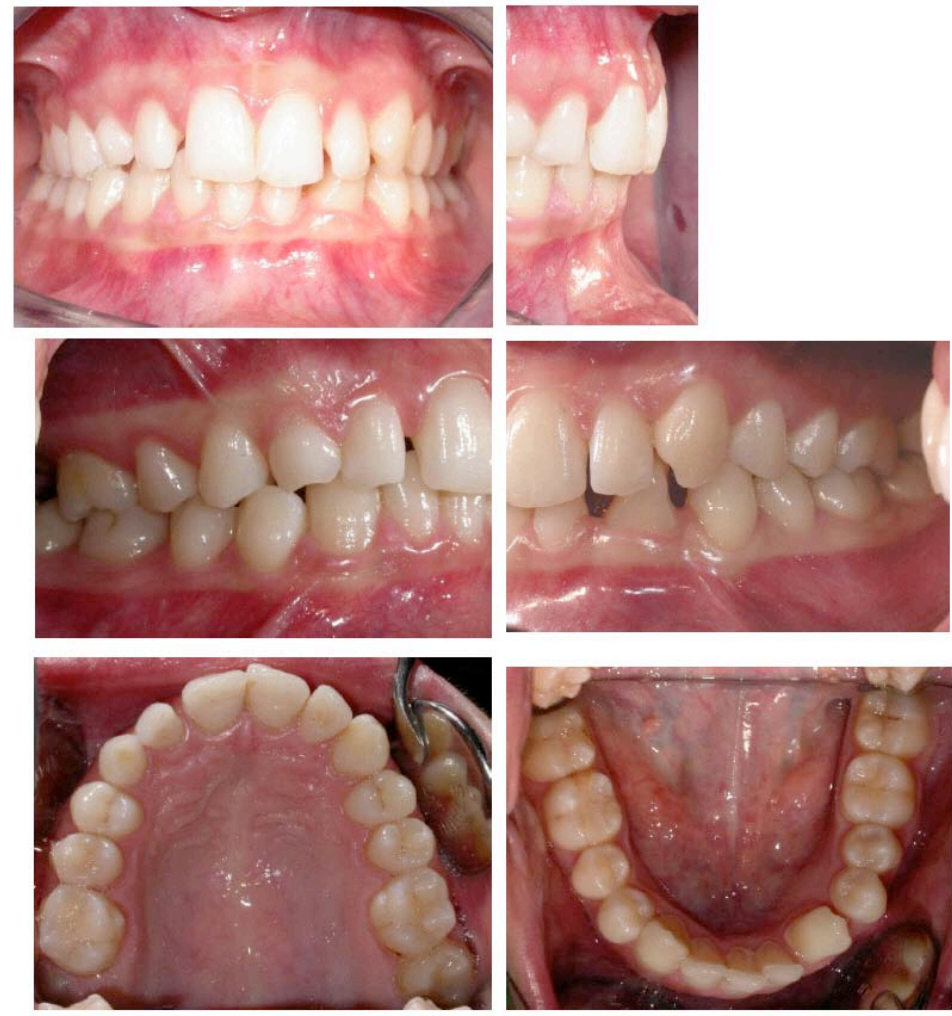

Fig. (2). Intraoral photographs before treatment. 

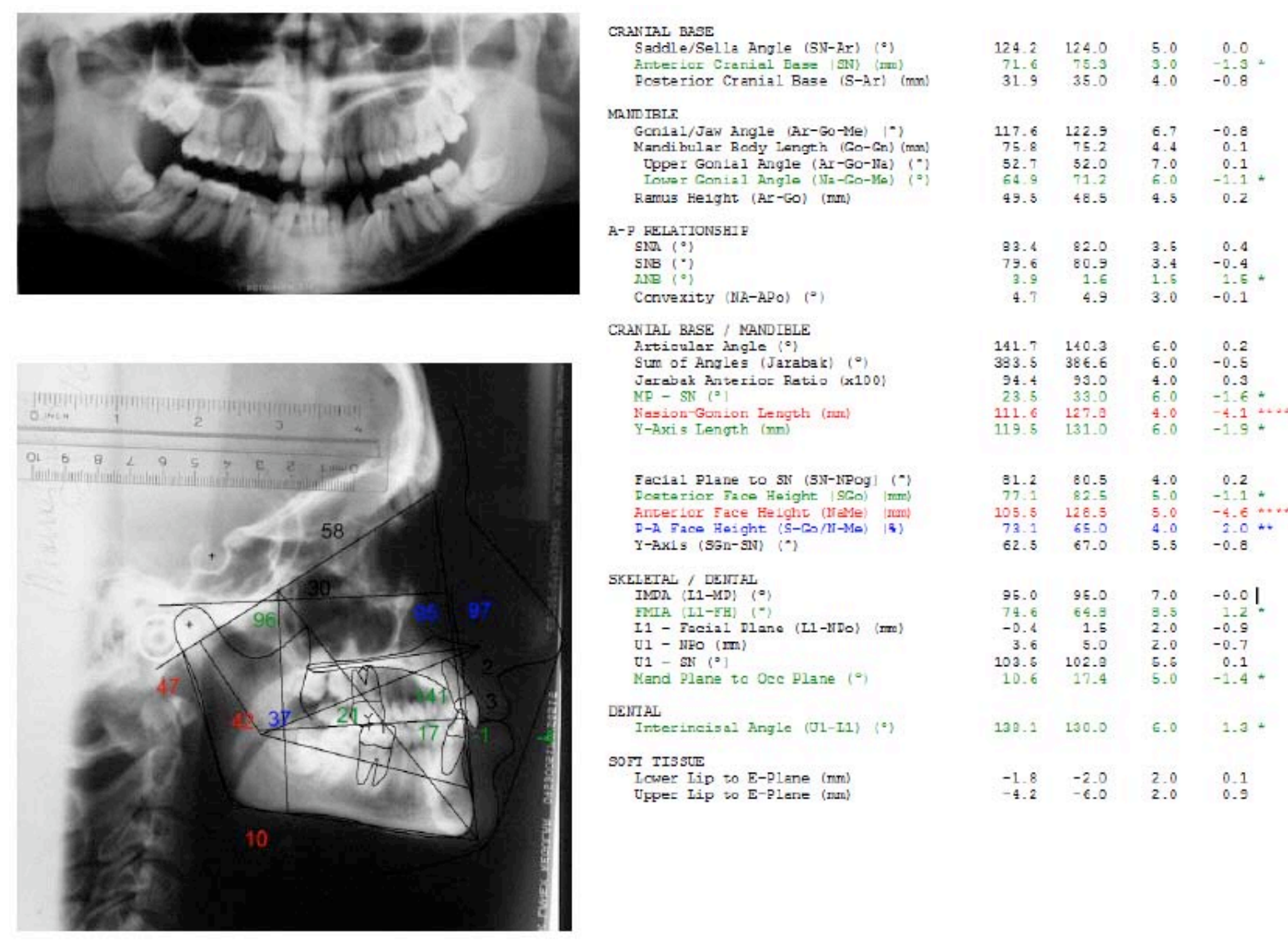

DEIIAL

Interincisal Angle (OI-I1) $\langle \%$

SOFT TISSTE

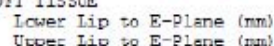

$\begin{array}{llll}130.1 & 130.0 & 6.0 & 1.3 . \\ -1.8 & -2.0 & 2.0 & 0.1 \\ -4.2 & -6.0 & 2.0 & 0.9\end{array}$

Fig. (3). Cephalometric values and panoramic radiograph at the start of treatment. From left to right: value measured, average value from the population norm, standard deviation of the average value from the population norm, difference from the extreme value of the population norm. Red: values above the norm, Green: values below the norm, Black: values within the norm.

The treatment plan included the orthodontic recovery of the upper maxillary canine and the lower tooth transposition.

The recovery of a maxillary impacted tooth would be performed preferably by using cantilever mechanics, along with a transpalatal arch, in order to improve dental arch anchorage, preserve arch form, and avoid effects from movements of adjacent teeth.

A fixed multibracket appliance was placed to align, level, and manage spacing of both upper and lower dental arches. Thermal Ni-TI archwires were preferred to increase patient compliance and reduce initial discomfort [10].

Initially, a .036 stainless steel transpalatal arch was modelled to obtain additional anchorage and to correct molar rotations. Alignment of both dental arches were achieved by using multibracket appliance (Roth prescription, slot size $.022 \times .028$ ) with heat activated Ni-Ti archwires (round .014 and round .016).The space for impacted and ectopic teeth was obtained by using superalastic coil springs on round .018 AJ Wilcock Australian wire (regular +, G\&H Orthodointics, Franklin, IN, US).

A flap for the surgical exposure of the palatal impacted maxillary right canine was obtained by an intrasulcular incision extended from the first left maxillary incisor to the second upper premolar of the same side. Once exposed, the palatal surface of the tooth was etched for 30 seconds and rinsed with water. Transbond XT (3M Unitek Monrovia, US) adhesive primer was used for its strength [11] following the instruction of the manufacturer. The lingual sheat was anchored to a .012 stainlees steel ligature, and bonded on to the distal palatal surface of the tooth, in order to prevent unwanted rotations during buccal movements. The ligature was modelled with eyelets distant $3 \mathrm{~mm}$ each other for cantilever insertion.

The cantilever is modelled using $.019 \times 025$ TMA (Ormco, Orange, Ca, US, Fig. 4).

The extrusion of the maxillary right canine was obtained by inserting a third order bend (read as a buccal root torque of the molar) at the insertion of the maxillary molar (Fig. 4). This activation lead to a caudal displacement of the hook at the boundary of the cantilever. The force delivered was about 150 grams. This cantilever was also activated to obtain a buccal movement of the tooth by activating the first order bend close to the molar.

To further obtain an adequate buccal root torque of the canine, a $-17^{\circ}$ torque bracket (second lower premolar) turned of $180^{\circ}$ was bonded.

The correction of tooth transposition was performed by using cantilever mechanics and powerchain for the control of 3.3 and 3.2. Two cantilevers were deisgned to extrude 3.2 and correct the rotation of 3.3 (Fig. 5). The space was than 


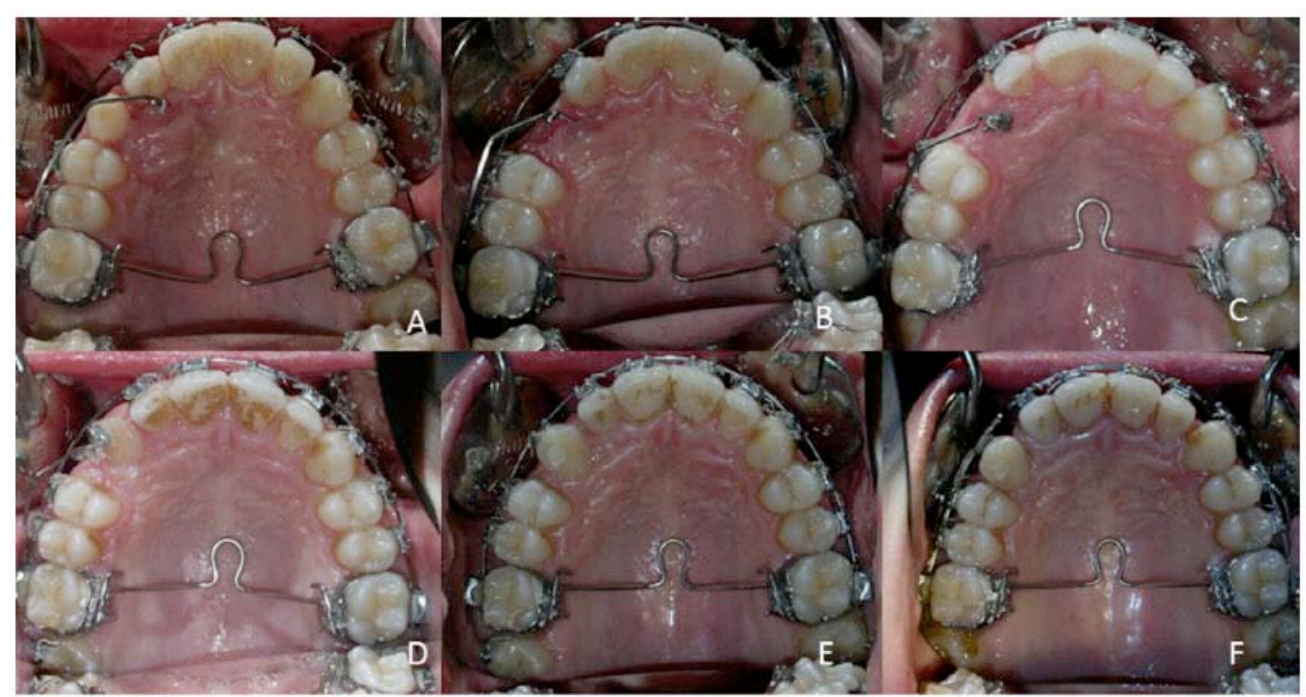

Fig. (4). Recovery of the impacted right maxillary canine. The effects of a 0.019x0.025 TMA Cantilever, activated for obtainind extrusion and labial movement are shown (A-D). Correction of canine torque (E-F).

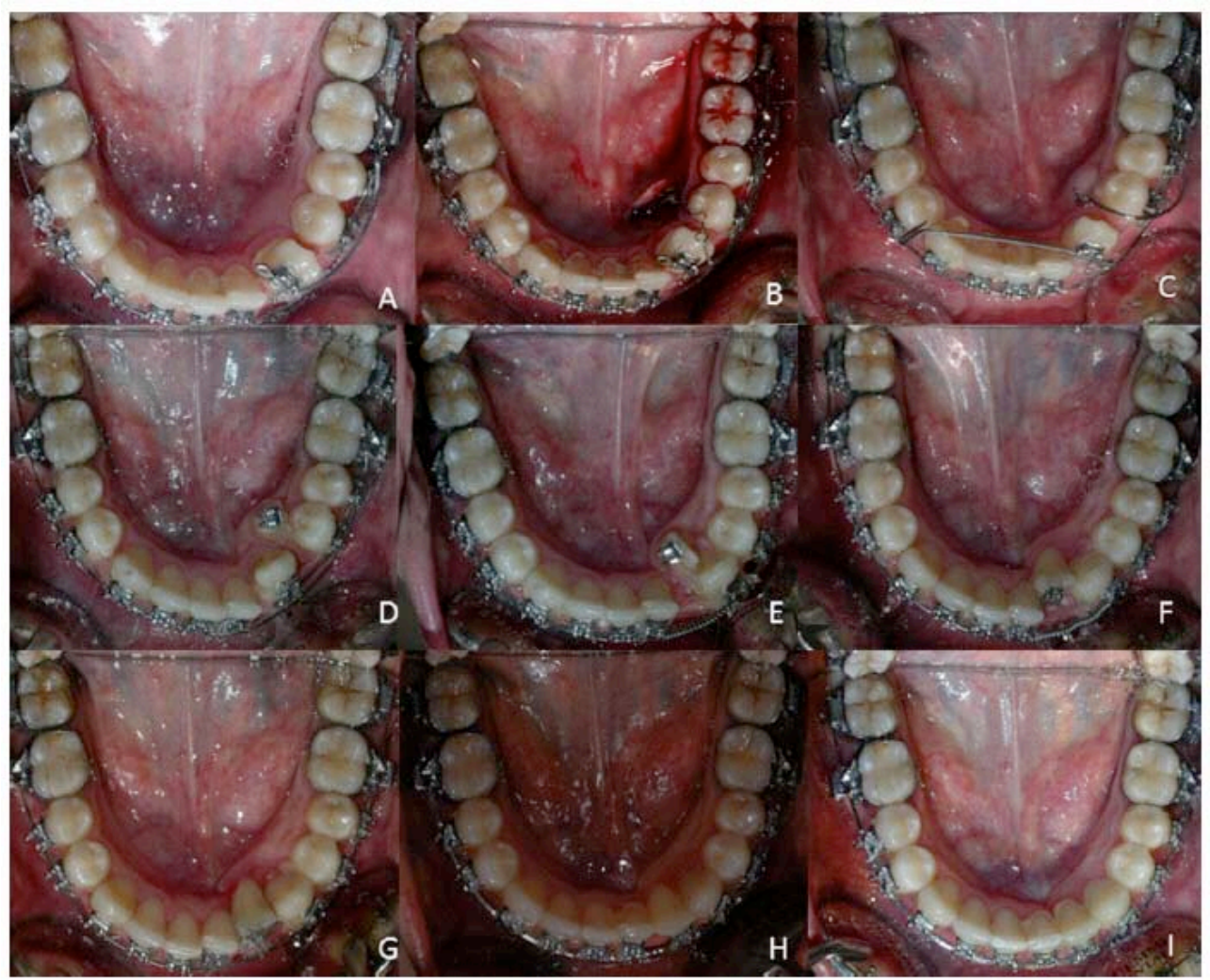

Fig. (5). Correction of tooth transposition. Cantilevers 0.019x0.025 (TMA, Ormco, Orange, Ca, US) were used. A) Cantilever inserted into 3.3 bracket slot and mesial to 4.6 to correct canine rotation. B) Impacted lower incisor was bonded and attached to a round superelastic archwire. C) Cantilevers for extrusion of 4.2 and for correction of canine rotation. D) Cantilever for intrusion of 4.3. E) Powerchain attached to lateral incisor to correct tooth rotation. Opel Ni-Ti coil spring is in place. F-G-H) The lower incisor is moved to its final position. I) Correction of torque of the lower incisor by using a -14 degrees torque bracket.

managed by using opel Ni-ti coil springs and powerchains. Additional labial root torque of the 3.2 was obtained by using a $17^{\circ}$ torque bracket (second lower premolar).

\section{Treatment Sequence}

Correction of first upper molar rotation with transpalatal arch
Upper and lower fixed straight wire self-ligating multi bracket appliances, with Dr. Roth prescription, slot .022;

- Finishing

_ Retention 

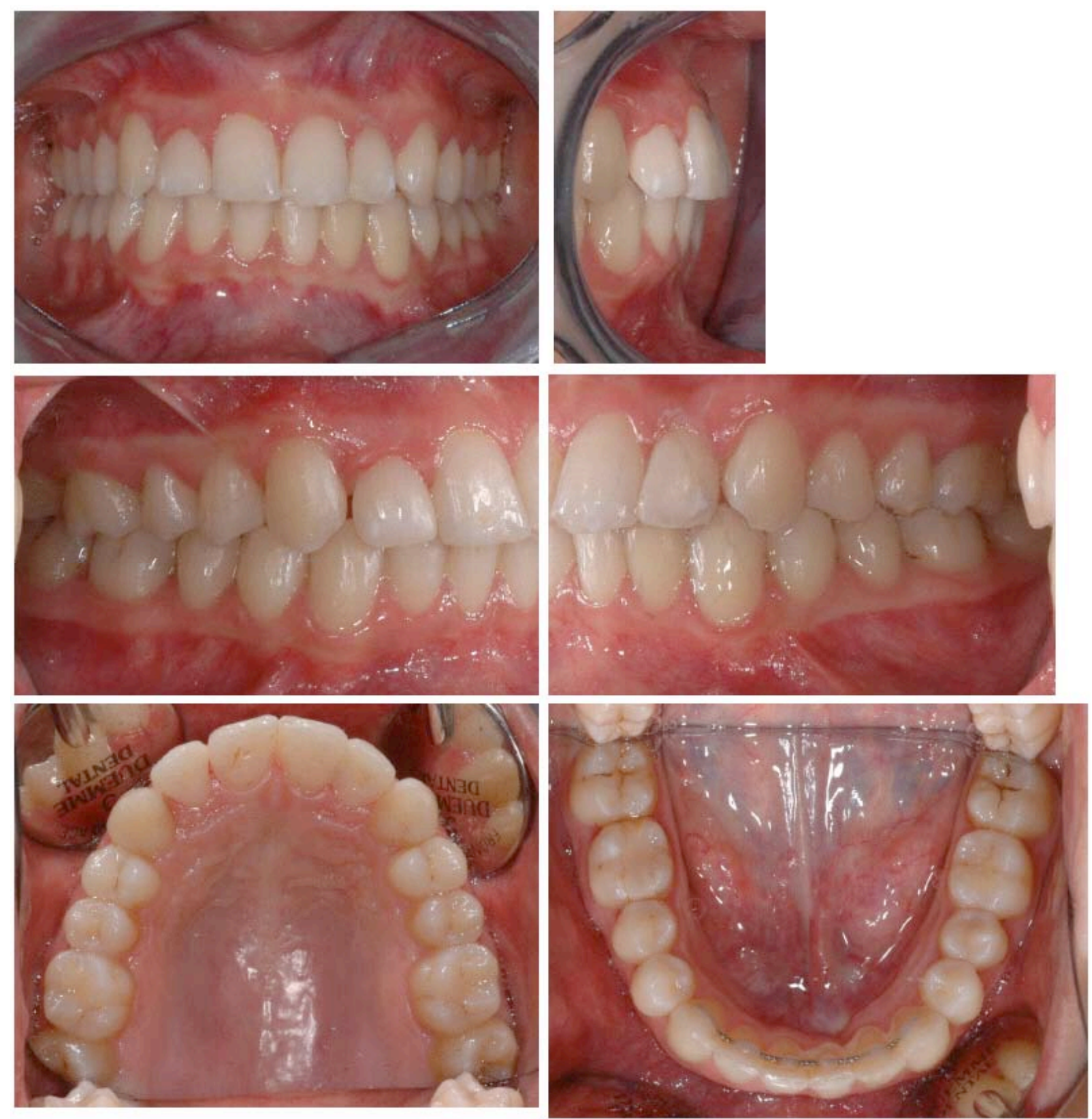

Fig. (6). Intraoral photographs after treatment.

\section{Archwire Selection}

-.014 HA Ni-Ti (heat-activated) upper and lower alignment archwires;

-.016 HA Ni-Ti upper and lower alignment archwires;

-.018 AJ Wilcock Australian regular plus (GH wire, Franklin, IN, US) upper and lower alignment archwires with coil open for space opening of the 4.2 and 1.3;

$-.018 \times .025 \mathrm{HA} \mathrm{Ni}$-TI wire upper and lower archwires;

$-.019 \times .025 \mathrm{SS}$ upper and lower archwires;

\section{Mechanics and Treatment Strategies}

- Use of Heat Activated Ni-Ti archwires to reduce orthodontic forces and improve patient discomfort [10].
- Cantilevers .019 x .025 (TMA, Ormco, Orange, Ca, US) for correction of tooth transposition.

-The upper incisors were retracted by a translation arch (.019 x .025 TMA, Ormco, Orange, Ca, US) [12].

-Finishing with $.018 \times .025$ multibraded wires with vertical elastics $(1 / 84 \mathrm{Oz})$.

-Retention with a lower retainer (wildcat round wire .0195, DENTSPLY GAC International, Islandia, NY, US) extended from 3.3 to 4.3 . The treatment duration was 28 months.

The intraoral and extraoral photographs after treatment are presented in Fig. (6) and Fig. (7). Cephalometric values after treatment and tracing superimpositions are shown in Fig. (8 and Fig. 9). 

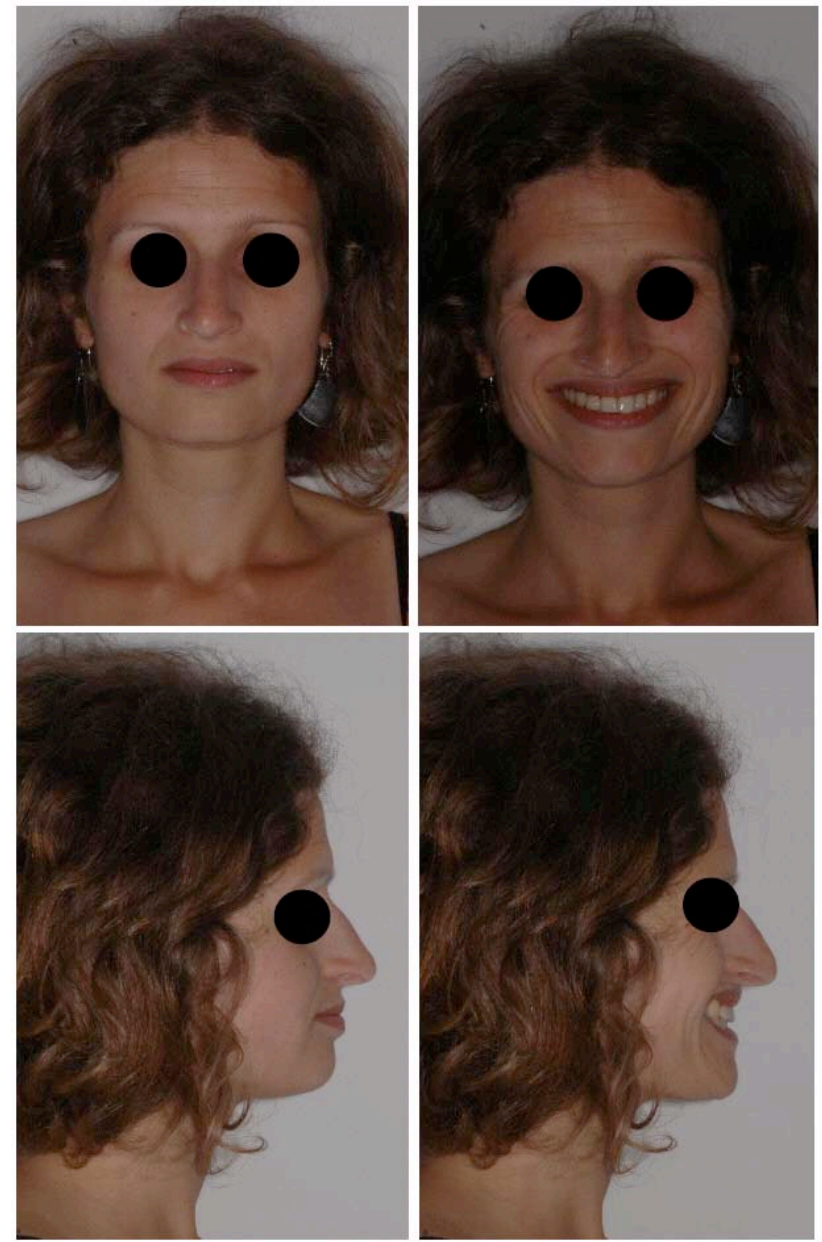

Fig. (7). Extraoral photographs after treatment.
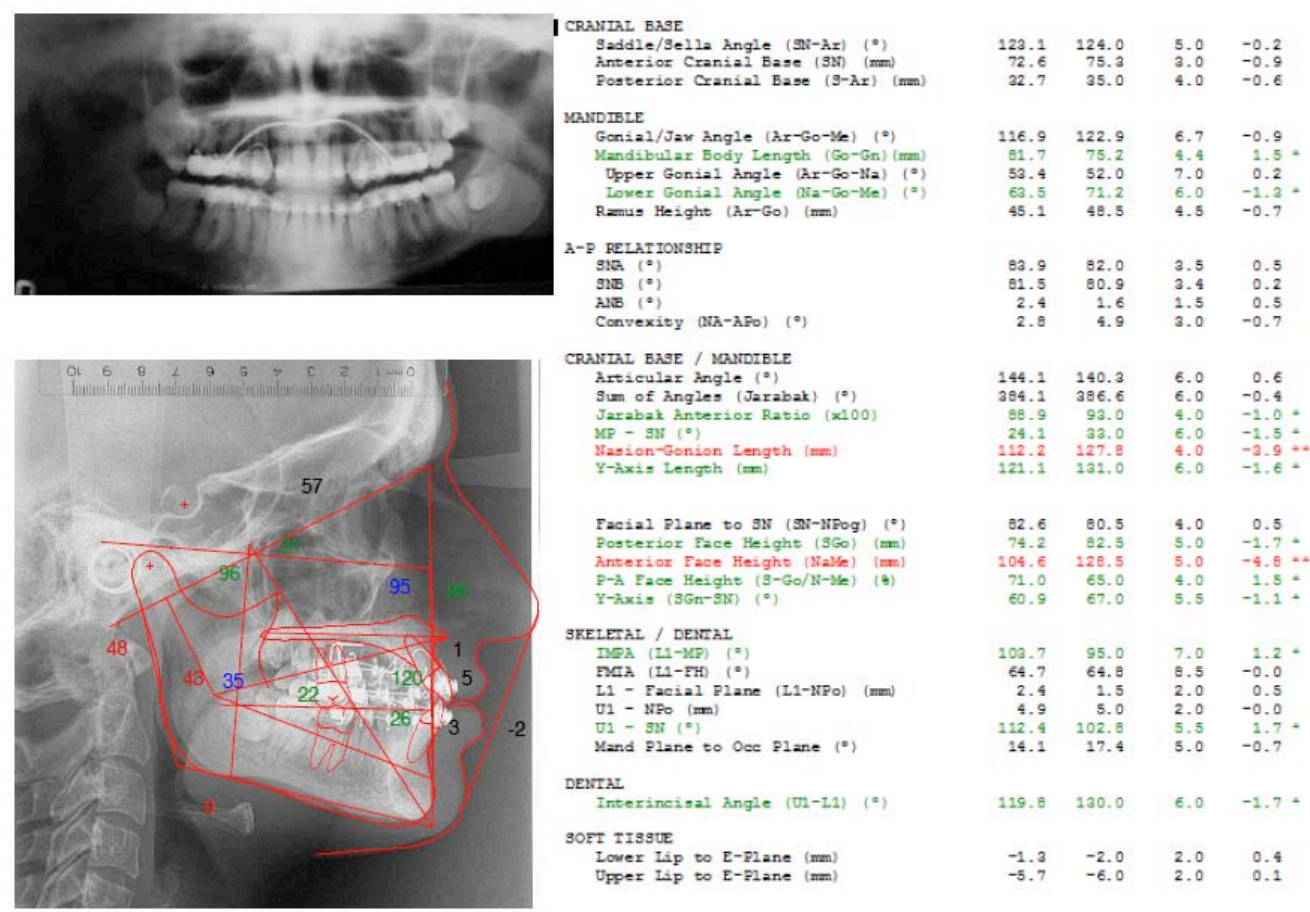

Fig. (8). Cephalmetric values and panoramic radiograph just before the end of treatment, before final debonding. From left to right: value measured, average value from the population norm, standard deviation of the average value from the population norm, difference from the extreme value of the population norm. Red: values above the norm, Green: values below the norm, Black: values within the norm 


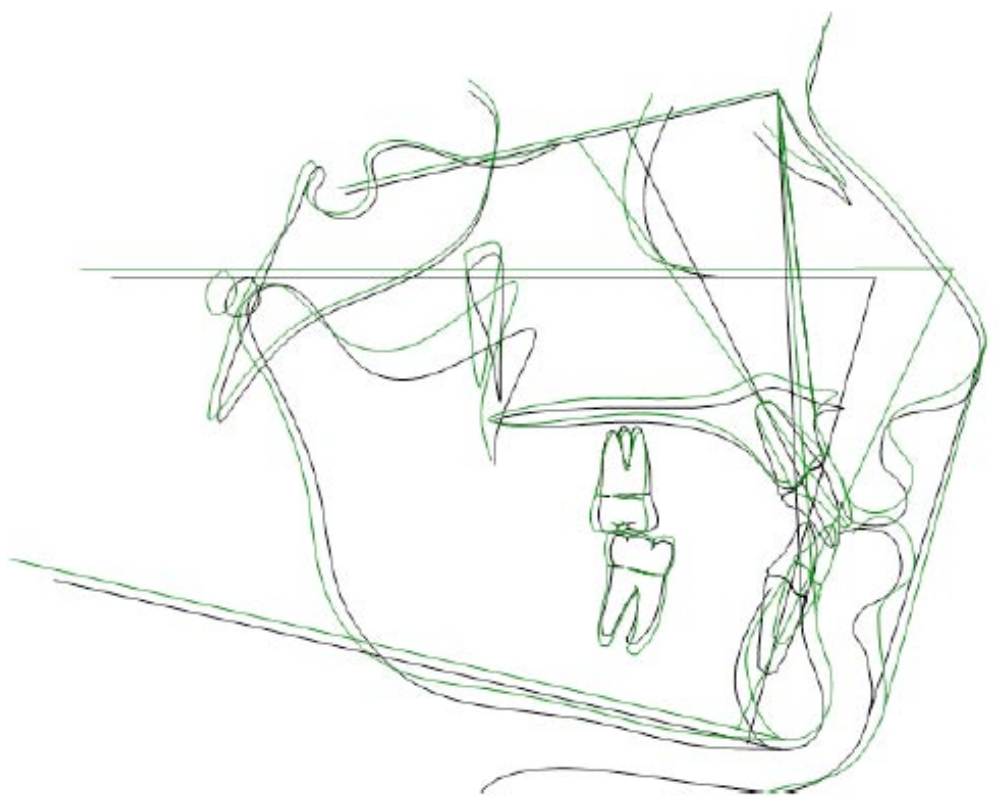

Fig. (9). Cephalometric tracing superimpositions. Black: before treatment. Green: end of treatment.

\section{CASE PRESENTATION - CASE 2 - WOMEN - 35 YEARS OLD}

\section{Diagnosis and Treatment Plan}

The extraoral and intraoral photographs of the patient are reported in Fig. (10). The patient presented this objective problem list:

- $\quad$ Angle Class I malocclusion;

- $\quad$ Retruded profile

- $\quad$ Presence of the deciduous maxillary canines, and impaction of both permanent maxillary canines

- $\quad$ Slight arch length discrepancies

- $\quad$ Severe brachifacial typology with reduced lower facial height with decreased overbite

Although she reported a whiplash occurred one year before, she did not present signs or symptoms of temporomandibular disorders [13]. Also, she was treated for a skeletal II malocclusion at the age of eleven by means of a Sander bite jumping appliance [14]. Nonetheless, she did not undergo a second phase treatment by fixed multibracket appliance.

\section{Treatment Sequence}

- Correction of first upper molar rotation with transpalatal arch

- $\quad$ Upper and lower fixed straight wire self-ligating multi bracket appliances, with Dr. Roth prescription, slot .022 ;

- $\quad$ Finishing

- Retention

\section{Archwire Selection}

-.014 HA Ni-Ti (heat-activated) upper and lower alignment archwires;
-.016 HA Ni-Ti upper and lower alignment archwires;

-.018 AJ Wilcock Australian regular plus (GH wire, Franklin, IN, US) upper alignment archwire with coil open for space opening of the 1.3 and 2.3;

-.018 x .025 HA Ni-TI wire upper and lower archwires;

-.019 x .025 SS upper and lower archwires;

\section{Mechanics and Treatment Strategies}

Cantilevers 0.019x0.025 (TMA, Ormco, Orange, Ca, US) were used to recovery the impacted canines, after space opening by open coil springs. They were firstly activated in order to extrude the teeth, and to obtain their distal movement. Then they were modelled to obtain a buccal movement. The anchorage was preserved by using a passive transpalatal arch, and stainless steel .019 x .025 archwire including the second maxillary molars. The activation of the cantilevers was scheduled about once a month. The rotation of the teeth was corrected by using elastomeric powerchains and .014 round $\mathrm{HA} \mathrm{Ni-Ti}$ archwires overlaying the stainless stell archwire.

The effects of the cantilevers are shown in Fig. (11). Post-treatment records are presented in Fig. (12). The treatment duration was 30 months.

\section{CASE PRESENTATION - CASE 3 - GIRL, 14 YEARS OLD}

\section{Diagnosis and Treatment Plan}

The extraoral and intraoral photographs of the patient are reported in Fig. (13). The patient presented this objective problem list: 

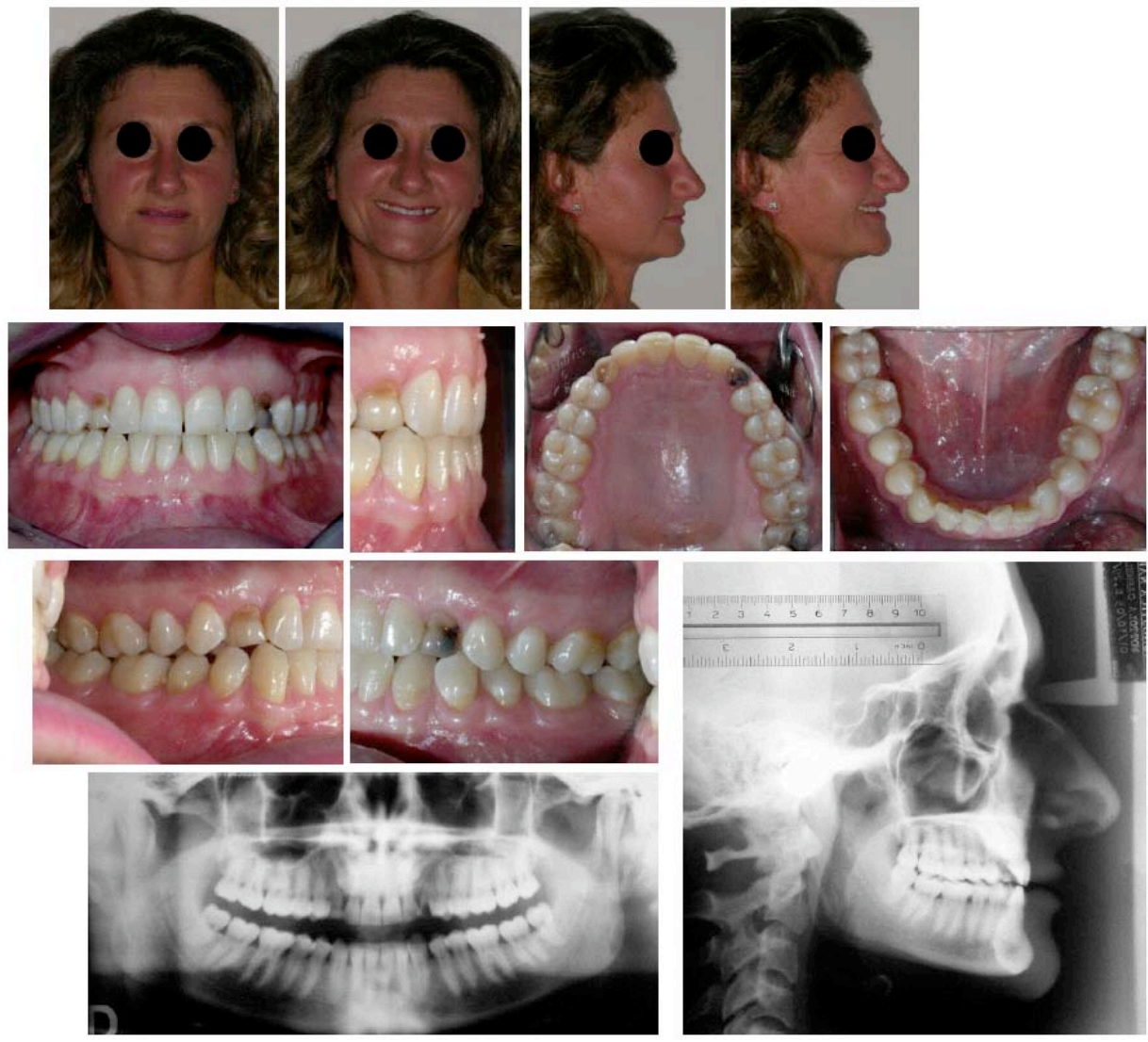

Fig. (10). CASE 2 - Pre-treatment records.
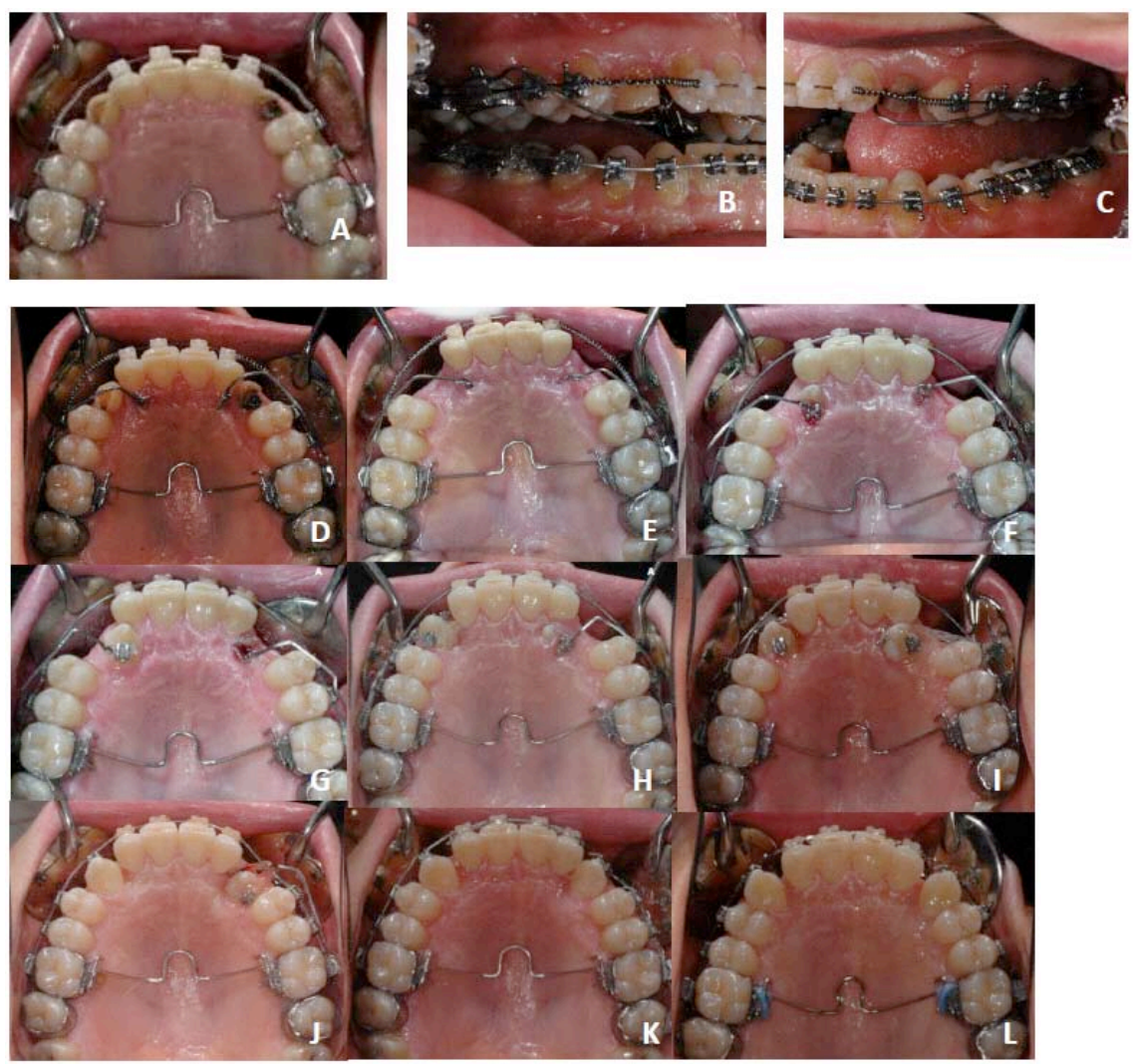

Fig. (11). CASE 2 - Cantilever in place (A-B-C). Progressive activation of the cantilever (D-J), correction of tooth rotation (D-E), correction of torque (K-L). 

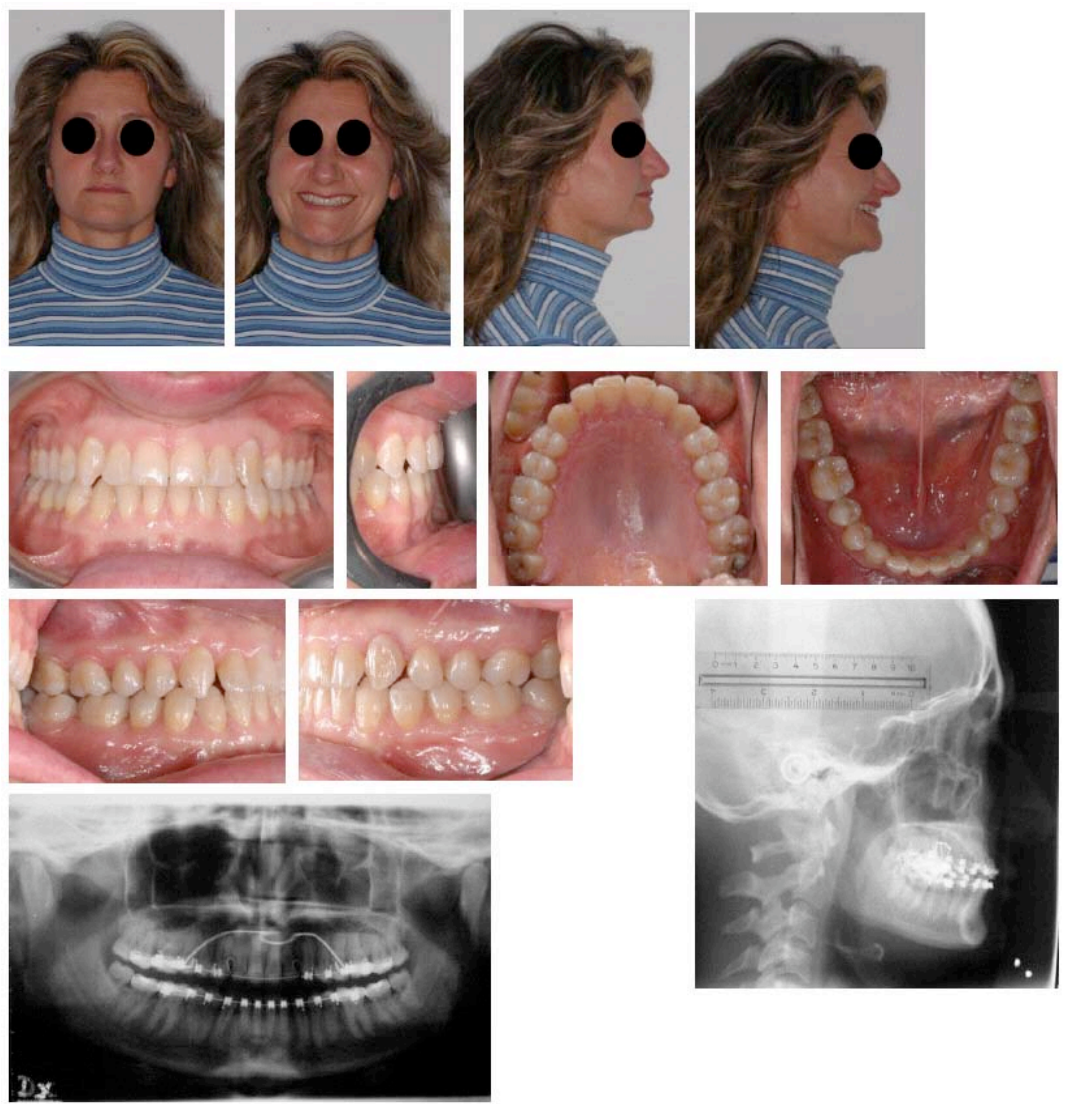

Fig. (12). CASE 2 Post-treatment records. Radiographs were collected before final debonding.
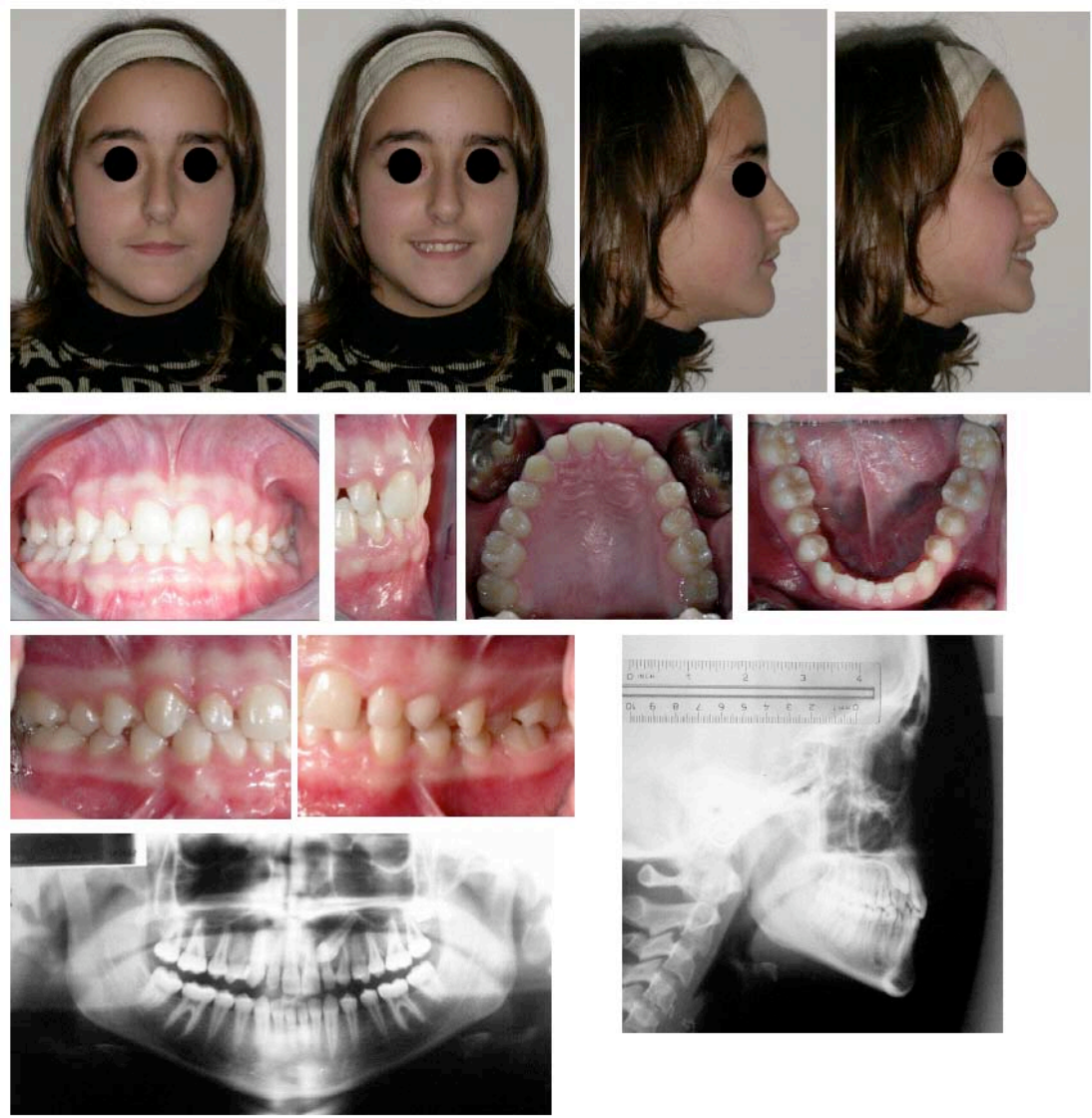

Fig. (13). CASE 3 - Pre-treatment records. 

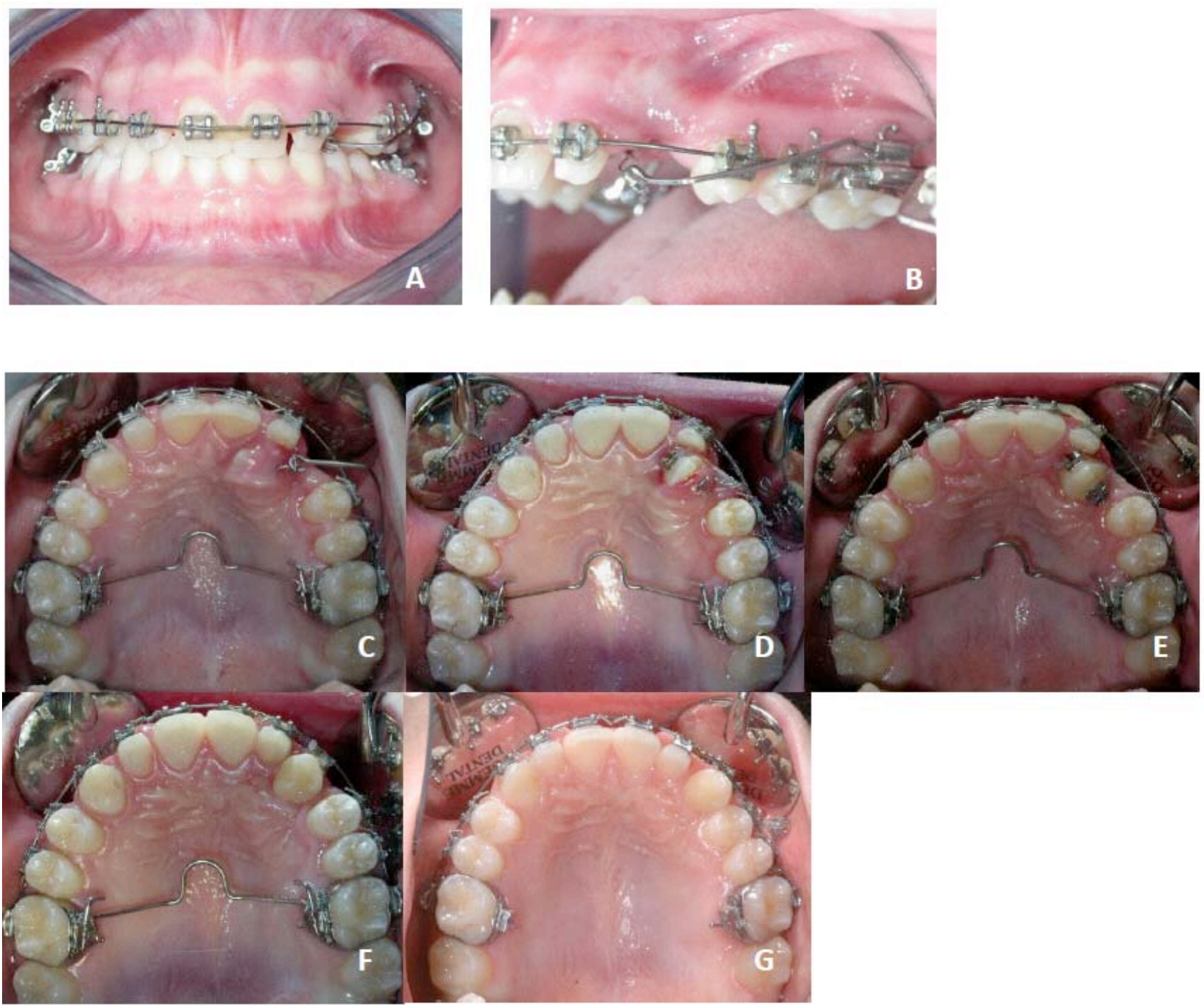

Fig. (14). CASE 3 - Cantilever in place (A-B). Progressive activation of the cantilever (C), correction of tooth rotation (D-E), correction of torque $(\mathrm{F}-\mathrm{G})$.

- Angle Class I malocclusion class III tendency;

- Retruded profile

- Presence of the left deciduous maxillary canine, and impaction of the permanent maxillary canine.

- Slight arch length discrepancies

Swe was treated at the age of 9 with a TPA proclination spring for correcting an anterior crossbite [15].

\section{Treatment Sequence}

- Correction of first upper molar rotation with transpalatal arch

- Upper and lower fixed straight wire self-ligating multi bracket appliances, with Dr. Roth prescription, slot .022 ;

- Finishing

- Retention

\section{Archwire Selection}

-.014 HA Ni-Ti (heat-activated) upper and lower alignment archwires;

-.016 HA Ni-Ti upper and lower alignment archwires;

-.018 AJ Wilcock Australian regular plus (GH wire, Franklin, IN, US) upper alignment archwire with coil open for space opening of the 2.3 ;

$-.018 \times .025 \mathrm{HA} \mathrm{Ni}$-TI wire upper and lower archwires;

$-0.019 \times 0.025$ SS upper and lower archwires;

\section{Mechanics and Treatment Strategies}

Also in this case, similar cantilevers were used to reposition the canine. Similar strategies (i.e. passive transpalatal arch, and stainless steel $0.019 \times 0.025$ archwire) were used to preserve anchorage.

The effects of the cantilevers are shown in Fig. (14). 

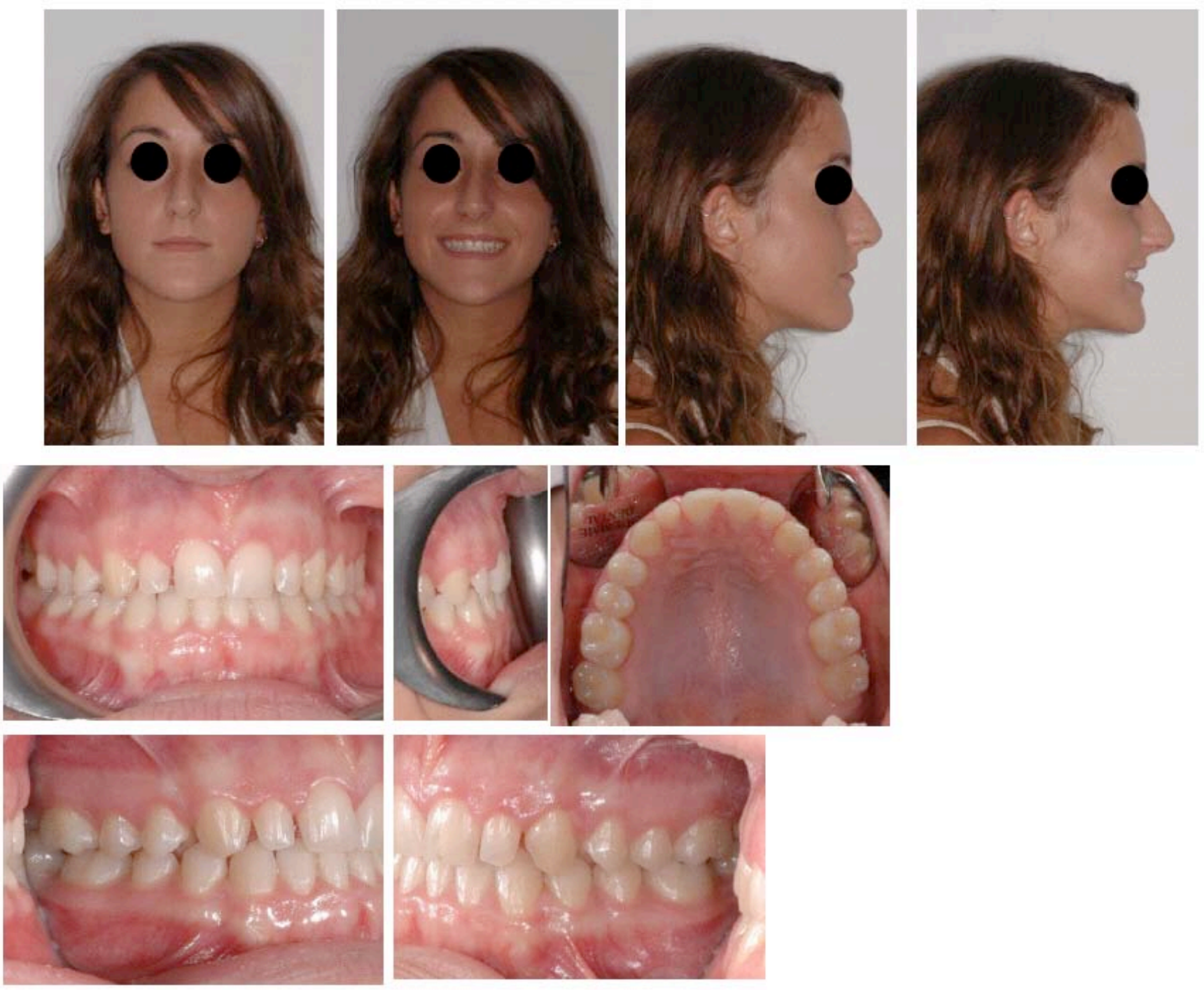

Fig. (15). CASE 3 - Post-treatment records.

Post-treatment records are presented in Fig. (15).

The treatment duration was 25 months.

\section{DISCUSSION}

In all the cases presented, the major treatment objectives were achieved.

The cantilever mechanics allowed a correct repositioning of the impacted teeth. However, since the force was applied on a single point of the canines, it did not allow for a proper correction of their torque [4]. Hence, in many cases, it was decided to use extratorque brackets for a proper torque control before finishing. From a clinical point of view, the use of cantilevers allowed an optimal control of the arch-forms, and determined a shorting of the treatment duration. Hence, it could be considered an efficient approach to manage cases with single or multiple impactions. It must be pointed up that the use of cantilevers may cause patient discomfort, at least after few days from the initial positioning. However, no breakages were reported by all the patients examined in this paper.
The major objective for the modelling of cantilevers are a correct activation, and the avoidance of tooth contact in occlusal relationship.

For all these orthodontic treatments we decided to use conventional brackets because it has been suggested that self-ligating brackets are critical for obtaining an adequate torque control [16]. Also we used heat activated Ni-Ti archwires to reduce patient discomfort [10] .

For all patients a retention period by means of a fixed lingual 3.3-4.3 retainer was scheduled. Good occlusal relationship and dental alignment were obtained in all cases.

\section{CONFLICT OF INTEREST}

The authors confirm that this article content has no conflicts of interest.

\section{ACKNOWLEDGEMENTS}

None declared. 


\section{REFERENCES}

[1] Yan B, Sun Z, Fields H, Wang L, Luo L. Etiologic factors for buccal and palatal maxillary canine impaction: a perspective based on cone-beam computed tomography analyses. Am J Orthod Dentofacial Orthop 2013; 143: 527-34.

[2] Bedoya MM, Park JH. A review of the diagnosis and management of impacted maxillary canines. J Am Dent Assoc 2009; 140:148593.

[3] Fischer TJ, Ziegler F, Lundberg C. Cantilever mechanics for treatment of impacted canines. J Clin Orthod 2000; 34: 647-50.

[4] Lindauer SJ, Isaacson RJ. One-couple orthodontic appliance systems. Semin Orthod 1995; 1: 12-24.

[5] Peck S, Peck L, Kataja M. Mandibular lateral incisor-canine transposition, concomitant dental anomalies, and genetic control. Angle Orthod 1998; 68: 455-66.

[6] Papadopoulos MA, Chatzoudi M, Kaklamanos EG. Prevalence of tooth transposition: a meta-analysis. Angle Orthod 2010; 80: 27585.

[7] Dworkin SF, LeResche L. Research diagnostic criteria for temporomandibular disorders: review, criteria, examinations and specifications, critique. J Craniomandib Disord 1992; 6: 301-55.

[8] Iodice G, Danzi G, Cimino R, Paduano S, Michelotti A. Association between posterior crossbite, masticatory muscle pain, and disc displacement: a systematic review. Eur J Orthod 2013; (in press).
[9] Michelotti A, Cioffi I, Festa P, Scala G, Farella M. Oral parafunctions as risk factors for diagnostic TMD subgroups. J Oral Rehabil 2010; 37: 157-62.

[10] Cioffi I, Piccolo A, Tagliaferri R, Paduano S, Galeotti A, Martina R. Pain perception following first orthodontic archwire placementthermoelastic $v s$ superelastic alloys: a randomized controlled trial. Quintessence Int 2012; 43: 61-9.

[11] Valletta R, Prisco D, De Santis R, Ambrosio L, Martina R. Evaluation of the debonding strength of orthodontic brackets using three different bonding systems. Eur J Orthod 2007; 29: 571-7.

[12] Martina R, Paduano S. The translation arch. J Clin Orthod 1997; 31: 750-3.

[13] Marini I, Paduano S, Bartolucci ML, Bortolotti F, Bonetti GA. The prevalence of temporomandibular disorders in patients with late whiplash syndrome who experience orofacial pain: a case-control series study. J Am Dent Assoc 2013; 144: 486-90.

[14] Martina R, Cioffi I, Galeotti A, et al. Efficacy of the Sander bitejumping appliance in growing patients with mandibular retrusion: a randomized controlled trial. Orthod Craniofac Res 2013; 16: 11626.

[15] Paduano S, Spagnuolo G, Biase Gd, Cioffi I. Treatment of a class II division patient with severe skeletal discrepancy by using a custom made TPA proclination spring. Open Dent J 2013; 7:109-17.

[16] Paduano S, Cioffi I, Iodice G, Rapuano A, Silva R. Time efficiency of self-ligating $v s$ conventional brackets in orthodontics: effect of appliances and ligating systems. Prog Orthod 2008; 9: 74-80.

(C) Paduano et al.; Licensee Bentham Open

This is an open access article licensed under the terms of the Creative Commons Attribution Non-Commercial License (http://creativecommons.org/licenses/by-nc/3.0/) which permits unrestricted, non-commercial use, distribution and reproduction in any medium, provided the work is properly cited. 